\title{
Usefulness of $\Phi$-order kinetics for the investigation, characterisation and quantification of photodegradation processes.
}

\author{
Mounir Maafi, Mohammed Alqarni \\ Leicester School of Pharmacy, De Montfort University, The Gateway, Leicester LE1 9BH, UK
}

\begin{abstract}
Ф-order kinetics has been shown to be the best tool to study photokinetics [ $1-6]$. Various species have been studied using this approach including photochromes $[1,2]$ and drugs $[3-6]$. The unimolecular photodegradation processes of the form $A B(1 \Phi)$ and photoreversible systems $A B$ (2Ф), where $A$, the initial species phototransforms into a product $B(A \rightarrow B)$ which itself can return to $A(A \rightarrow B)$ via two distinct photoprocesses, each characterized each by an individual efficiency $(\Phi)$, have been mathematically described by integrated rate-law equations which express the so called ' $\Phi$-order' kinetics [1 -6]. The application of this novel approach was found to be useful to determine a number of photoreaction attributes. The reaction's photochemical quantum yield(s), the effects of initial species concentration, or the impact of competitive absorbers on the rate-constant of the photoactive species were all made readily accessible by simple methods. More importantly, the $\Phi$ order kinetics has proven to facilitate the development of a wide range of actinometers from $A B$ $(1 \Phi)$ and $A B(2 \Phi)$ systems [2 -6]. This novel $\Phi$-order kinetic method clearly has the potential for application in various fields including the photodegradation of natural products. The details of $\Phi$ order elucidation kinetic methods will be discussed here and examples of application presented.
\end{abstract}

Keywords: Spectrophotokinetics, photodegradation; $\Phi$-order kinetics; quantum yield; actinometry; UV/Vis spectroscopy

Acknowledgements: The authors would like to thank Taif University, Saudi Arabia and the Saudi cultural Bureau (in London), for their help, assistance and financial support.

Keywords: Spectrophotokinetics, photodegradation; $\Phi$-order kinetics; quantum yield; actinometry; UV/Vis spectroscopy.

References:

[1] Maafi M, Brown RG. The kinetic model for AB (1 Ф) systems: A closed-form integration of the differential equation with a variable photokinetic factor. J Photochem Photobiol A: Chemistry 2007; 187: $319-324$.

[2] Maafi M. The potential of AB (1Ф) systems for direct actinometry. Diarylethenes as successful actinometers for the visible range. Phys Chem Chem Phys 201012: 13248 13254. 
[3] Maafi W, Maafi M. Modelling nifedipine photodegradation, photostability and actinometric properties. International journal of pharmaceutics. 2013;456(1):153 - 64 .

[4] Maafi M, Maafi W. Ф-order kinetics of photoreversible-drug reactions. Int J Pharm. 2014; 471: $536-543$.

[5] Maafi M, Maafi W. Montelukast photodegradation: Elucidation of $\Phi$-order kinetics, determination of quantum yields and application to actinometry. Int J Pharm 2014; 471: 544 -552 .

[6] Maafi M, Lee LY. Determination of Dacarbazine $\Phi$-Order Photokinetics, Quantum Yields, and Potential for Actinometry. J Pharm Sci 2015; 104: 3501 - 3509. 